\title{
Soluble NKG2D Ligands Impair CD8+ T Cell Anti- Tumor Function Dependent Of NKG2D Downregulation In The Neuroblastoma
}

\section{Yi Zhang}

Children's Hospital of Fudan University

Feifei Luo ( $\nabla$ feifeiluo@fudan.edu.cn )

Fudan University

Kuiran Dong

Children's Hospital of Fudan University

\section{Research Article}

Keywords: Neuroblastoma, NKG2D receptor, soluble NKG2DL, ADAMs

Posted Date: February 25th, 2022

DOI: https://doi.org/10.21203/rs.3.rs-1387262/v1

License: (c) (1) This work is licensed under a Creative Commons Attribution 4.0 International License.

Read Full License 


\section{Abstract}

T cell-based immunotherapy has achieved remarkable clinical outcomes, but it is still restrained by the tumor-induced immunosuppression. Tumor-derived NKG2D ligands are one of the immunosuppression factors, which allow tumor cells to escape from the immunologic surveillance. However, the underlying mechanisms of NKG2D ligands (NKG2DL)-mediated immune evasion are not fully understood, especially in neuroblastoma. In this study, we firstly found the significant up-regulation of the soluble NKG2DL sMICA/B and sULBP2 in the serum of neuroblastoma patients, which was mainly derived from neuroblastoma tumor cells and shed by ADAM10 and ADAM17. Furthermore, sNKG2DLs expression levels were negatively correlated with the immunosuppressive microenvironment and poor prognosis. Tumor-derived sNKG2DL induced degradation of NKG2D on $C D 8^{+} \mathrm{T}$ cells and impaired $\mathrm{CD} 8^{+} \mathrm{T}$ cell proliferation, IFN-y production, and CD107a translocation. More importantly, the blockade of tumorderived sNKG2DLs recuperates anti-tumor activity of $\mathrm{CD} 8^{+} \mathrm{T}$ cell. Thus, our data uncover a novel neuroblastoma-induced immunosuppression, and offer a promising strategy to recover T-cell function and enhance anti-tumor immunotherapy.

\section{Introduction}

Neuroblastoma (NB) is the most common extracranial tumor diagnosed in children. Widely accepted standard therapy [1] for high-risk NB includes 5 to 7 cycles of intensive cytotoxic chemotherapy, surgery, consolidative autologous stem cell transplantation, radiation therapy, and maintenance immunotherapy with anti-GD2 antibodies [2]. These standard therapies carry considerable toxicity while survival remains generally poor as NB accounts for $15 \%$ of deaths attributable to cancer in childhood [3]. Novel therapeutic approaches are eagerly needed for NB.

NKG2D is an activating receptor (transmembrane protein) belonging to the NKG2 family of c-type lectinlike receptors [4]. It usually expresses on the surface of natural killer (NK) cells, all CD8 ${ }^{+} \mathrm{T}$ cells, as well as certain activated $C D 4^{+}$T cells $[5,6]$. NKG2D acts as a primary activating receptor in NK cells, thus resulting in the activation of cellular cytotoxicity, cytokine secretion, and proliferation [7]. In T cell, NKG2D is a potent co-stimulator of TCR-mediated effector functions and up-regulates antigen-specific T cellmediated cytotoxicity directed against cells or tissues expressing stress-induced NKG2D ligands (NKG2DLs), particularly under conditions of suboptimal TCR engagement [8]. The NKG2DLs include MHC class I chain-related protein A and B (MICA and MICB), and the cytomegalovirus-related UL-16 binding proteins1-5 (ULBP1-5) [9], which usually express on "stressed" cells as infected or malignant cells. They are all membrane-bound glycoproteins, distantly related to MHC class I molecules, and under certain circumstances they can be shed [10]. It has been found that NB could downregulate NKG2D ligands to prevent themselves from the recognition and elimination by NK cells [11]. Meanwhile, NKG2DLs released by NB cells could internalize NKG2D receptors on the surface of NK or T cells, and thereby impair NK or T cell-mediated anti-tumor immunity [12]. 
The use of immune cells engineered with the proposed chimeric antigen receptor (CAR) design are new promising approaches of adoptive cell therapy in NB [13]. Two clinical studies have been conducted with first generation CAR-T cells in patients with NB $[14,15]$. CAR-NK cells also have been generated and achieved increased killing of tumor cell lines in vitro [16]. Indeed, the success of CAR-T/NK-cell therapy in solid tumors relies on the ability of CAR-engineering cells to enter in the tumor site, overcome the immunosuppressive tumor microenvironment, and persist for a long period. However, the clinical efficacy of these immune cells in NB tumors would be inhibited by immune suppressive conditions of NKG2DLs release.

In the present work, we found high levels of soluble NKG2DLs in the sera of NB patients, which were closely associated with poor outcome and T cell exhaustion. And these tumor-derived NKG2DLs were shed by a disintegrin and metalloproteases (ADAM) 10 and ADAM 17. Then, they impaired the proliferation, IFN-y production, and cytotoxicity of $\mathrm{CD} 8^{+} \mathrm{T}$ cells through NKG2D receptor. The blockade of these tumor-derived sNKG2DLs could recuperate anti-tumor function of CD8 ${ }^{+} \mathrm{T}$ cells, which may supply an attractive strategy for improving T cell-mediated immunotherapy.

\section{Material And Methods}

\section{Patient samples}

Peripheral blood were collected from stage IV NB patients undergoing standard treatments (surgery and chemotherapy) at the Children's hospital of Fudan University according to the protocols approved by the ethics committee of Fudan University. Peripheral blood was equally obtained from patients with benign pediatric surgical disease, e.g., oblique inguinal hernia, and further referred to as normal controls.

Mononuclear cells were derived from ascites and peripheral blood, isolated by Lymphocyte Separation Medium 1077 (PromoCell), and cryopreserved or freshly used for immunophenotyping. Cell-free ascites and plasma was preserved for analysis of soluble NKG2D ligands.

The collection and the analysis of human material were approved by the local ethics committee of the Fudan University.

\section{Cell Culture}

The purity of the isolated T cell subpopulations was $>95 \%$ as assessed by flow cytometry analysis. $T$ lymphocytes were re-suspended at $1 \times 10^{6}$ cells per $\mathrm{mL}$ in complete medium and cultured in 24 well plates with $5 \mu \mathrm{g} / \mathrm{mL}$ anti-CD3 (pre-coated), $2 \mu \mathrm{g} / \mathrm{mL}$ anti-CD28 and $10 \mathrm{U} / \mathrm{mL}$ IL-2. Then, $T$ cells were harvested, washed twice with PBS, counted, detected and used for coculture.

SH-SY-5Y, SK-N-BE (2) and SK-N-SH cells were purchased from the Cell Bank of the Chinese Academy of Science (Shanghai, China). CHLA-15 and LA-N-5 cells were gifts from Professor Mujie Ye of Fudan 
University. All cells were cultured in DMEM and F12 $(1: 1)$ with $10 \%$ fetal bovine serum in a humidified incubator with $5 \% \mathrm{CO}_{2}$ at $37^{\circ} \mathrm{C}$. These cell lines were exposed for $24 \mathrm{~h}$ to the ADAM10 inhibitor GI254023X, or ADAM 17 inhibitor TAPI-1. ADAMs promotor PMA was added for 40 min before collecting SN and tumor cell lines. GI254023X, TAPI-1 and PMA are purchased from ApexBio company.

\section{Flow Cytometry}

NB tumor cells $\left(1 \times 10^{6}\right)$ were stained with directly conjugated Abs: MICA/B, ULBP1-3 (eBioscience, USA) and ADAM10, ADAM17 (abcam, USA) or with appropriate isotype-matched control IgG (eBioscience). T cells were stained for surface markers CD8, CD4, CD25, CD45R0, CCR7, NKG2D, CD107a or intracellular marker Foxp3, IFN-y, Ki67 with fluorescence-conjugated Abs according to the manufacturer's instructions (eBiosciences). For flow cytometry, cells were counted and incubated with Fc blocker for 30 min, followed by 30-min incubation with conjugated antibodies for surface markers. For intracellular cytokine detection, cells were stimulated in vitro with Cell Stimulation Cocktail (eBiosciences) for $5 \mathrm{~h}$ at $37^{\circ} \mathrm{C}$ before FACS analysis. After stimulation, cells were stained for surface markers and cytokines with Intracellular Fixation and Permeabilization Buffer Set (eBiosciences).

\section{ELISA}

The concentrations of soluble MICA/B and ULBP1-3 in serum and culture supernatant of tumor cells were quantified by ELISA following the manufacturer's instructions (Thermo Fisher, USA).

\section{Statistical analysis}

Data are presented as mean \pm SEM. Analyses were done with GraphPad Prism version 6.0 (Graphpad Software, Inc., San Diego, CA). Data were analyzed with Student's $t$-test, Mann-Whitney nonparametric test, one-way ANOVA, two-way ANOVA, or log-rank test as indicated. The statistical significance level was

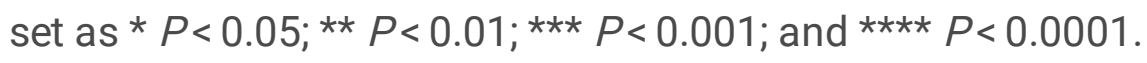

\section{Results}

\section{Tumor-derived sNKG2DLs are shed by ADAM10 and ADAM17}

In order to investigate the role of NKG2DLs, we firstly measured the expression of the soluble NKG2DLs (MICA/B and ULBP) in serum samples from 35 NB patients and 10 healthy donors by ELISA. sMICA/B in serum from NB patients was $269.2 \pm 88.3 \mathrm{pg} / \mathrm{mL}$ compared with $3.94 \pm 0.73 \mathrm{pg} / \mathrm{mL}$ in healthy donors (Fig. 1A). sULBP-2 was $42.61 \pm 13.63$ compared with $4.69 \pm 0.87$ in healthy donors (Fig. 1A). The differences between the concentrations of sMICA/B and SULBP-2 in sera from NB patients and healthy donors were statistically significant $(P<0.001)$, as determined by Mann-Whitney nonparametric test (Fig. 1A). Consistently, the expressions of MICA/B and ULBP 1-3 could be detected on NB cell lines, 
including SH-Y5Y, CHLA-15, SK-N-BE (2), LA-N-5, and SK-N-SH using flow cytometry (Fig. 1B). Also, the presence of sMICA/B and sULBP 1-3 was evaluated in culture supernatants (SN) of NB cell lines by ELISA. High levels of SMICA/B, sULBP-2 and sULBP-3 were detected in SN of SH-Y5Y, SK-N-BE (2), LA-N-5, after 48 hours culture (Fig. 1C). sULBP-1 showed low expression level in all cell lines (Fig. 1C). Meanwhile, all the NB cells analyzed showed surface expression of ADAM10 and ADAM17 evidenced by immunofluorescence with the specific mAb and FACS analysis (Fig. 1D). To evaluate whether ADAM10 and ADAM17 would be associated with sNKG2DLs releasing, NB cells were exposure to selective ADAM10 or ADAM17 promoters and inhibitors. Soluble NKG2DLs (sMICA/B, sULBP2, sULBP3) were measured by ELISA in the SN collected from NB cell line exposed for $24 \mathrm{~h}$ to the ADAM10 inhibitor GI-X (from $10 \mu \mathrm{M}$ to $40 \mu \mathrm{M}$ ), ADAM 17 inhibitor TAPI-1 (from $15 \mu \mathrm{M}$ to $60 \mu \mathrm{M}$ ), or the solvent alone (DMSO). ADAMs promotor PMA was added for 40 min before collecting $S N$ and tumor cell line. Cell surface expression of NKG2DLs (MICA/B, ULPB2, ULBP3) was analyzed by FACS analysis. PMA could enhance the enzymatic effect of ADAM10 and ADAM17, induce releasing of soluble NKG2DLs in SN and shielding NKG2DLs on NB cell line surface (Fig. 1E, G). Combined ADAM10 inhibitor and ADAM 17 inhibitor could inhibit shedding of NKG2DLs and increase its cell surface expression in NB cell line with a concentration dependent effect (Fig. 1F, G). Neither ADAM10 inhibitors GI-X nor ADAM 17 inhibitors TAPI-1 could produce similar effects (Fig. $1 F)$. Thus, the production of SNKG2DL is a characteristic of primary neuroblastomas and neuroblastoma cell lines, and these tumor-derived sNKG2DLs are shed by ADAM10 and ADAM17.

sNKG2DLs are negatively correlated with patient prognosis and the low-exhausted phenotype of T cells.

Also, we collected serum samples of patients at time points of pre and post standard treatments (surgery and chemotherapy), and then detected both SMICA/B and sULBP-2 levels. Compared with the preoperative and subtotal resection (STR) groups, SMICA/B and sULBP-2 levels significantly decreased in gross total resection (GTR) group (Fig. 2A), indicating the expression of MICA/B and ULBP-2 might be negatively correlated with patient prognosis. Next, given that both MICA/B and ULBP-2 were immunerelated molecules, we assessed whether the decreased expression of sULBP2 and SMICA/B would affect the immune pattern of patients. PBMCs were isolated from patients after standard treatment and stained with different immunophenotypic markers. There was no change in the percentages of regulatory $T$ cell (Treg), B cells, NK cell and NKT lymphocyte subpopulations between GTR and STR groups (Fig. 2B, data not shown). But a significantly decreased percentage in $\mathrm{CD}^{+} \mathrm{T}$ cells was shown in STR group than that in GTR group (Fig. 2B). On further analysis of IFN- $\gamma^{+} \mathrm{CD} 4^{+}$and IFN- $\gamma^{+} \mathrm{CD} 8^{+} \mathrm{T}$ cell subpopulation, percentages in both PD $-1^{+}$, and $\mathrm{Tim}-3^{+}$were significantly increased in STR group, compared with GTR group (Fig. 2C). These observations imply that the down-regulation of sULBP2 and sMICA/B accompanies with the up-regulated frequency of T cells with low-exhausted phenotype.

\section{Snkg2dls Impair Cd8 T Cell Function And Memory Formation}


NKG2D expressed on activated NK, NKT and $C D 8^{+}$(less on CD4 $4^{+}$) T cell in human [10]. To evaluate whether soluble NKG2D ligands separately affected $C D 8^{+} T$ would be associated with NKG2D receptor, NKG2D expression was analyzed by flow cytometry. After 12-hour culture with different concentrated tumor cell supernatants, NKG2D down-modulation was observed on $\mathrm{CD}^{+} \mathrm{T}$ cell (Fig. 3A). And the degree of receptor down-modulation was associated with supernatants concentration (Fig. 3A). To study whether NKG2D down-modulation on $\mathrm{CD}^{+} \mathrm{T}$ cells had functional consequences, we tested human $\mathrm{T}$ cell function after culture with different concentrated supernatants of NB cell line for $12 \mathrm{~h}$. When CD8 ${ }^{+}$and $\mathrm{CD} 4^{+} \mathrm{T}$ lymphocytes were separately detected, we observed that sNKG2DL was selectively downregulate $\mathrm{CD}^{+} \mathrm{T}$ cells function, including proliferation, IFN- $\gamma$ production and cytotoxicity (Fig. 3B). Percentages of IFN- $\gamma^{+}, \mathrm{Ki}^{+} 7^{+}$and $\mathrm{CD} 107 \mathrm{a}^{+}$in $\mathrm{CD} 8^{+} \mathrm{T}$ cell were significantly decrease, particularly at high concentrated tumor culture supernatants (Fig. 3B).

NKG2D receptor had been defined as a costimulatory molecule in $\mathrm{CD} 8^{+} \mathrm{T}$ cells, and was responsible for $\mathrm{CD}^{+}{ }^{+}$T-cell memory formation[8]. We investigated whether soluble NKG2DL co-cultured T cells could reduce effector functions and memory formation. Cytofluorimetric analysis of the early $\left(C D 69^{+}\right)$and late activation markers (CD25+) expression on T cells was performed in soluble NKG2DL co-cultured T cells. The memory profiling of co-cultured T cell subsets was based on CD45RO and CCR7 expression.

Percentage of activated $\mathrm{CD} 8^{+} \mathrm{T}$ cell $\left(\mathrm{CD} 69^{+} \mathrm{CD} 25^{+}\right)$was significant decreased at high concentrated tumor cell supernatants (Fig. 3C), indicating the inverse correlation of tumor cell supernatant concentration with $\mathrm{CD} 8^{+} \mathrm{T}$ cell activation. A similar trend was seen in memory formation of $\mathrm{CD} 8^{+} \mathrm{T}$ cells. Differenced concentrated tumor cell supernatants induced decreased frequency of $\mathrm{CD} 45 \mathrm{RO}^{+} \mathrm{CCR} 7^{+}$memory $\mathrm{CD} 8^{+} \mathrm{T}$ cells (Fig. 3D). While there was no significant correlation of $C D 4^{+} \mathrm{T}$ cell subset activation and memory profiling. Taken together, sNKG2DLs impair $\mathrm{CD} 8^{+} \mathrm{T}$ cell activation, proliferation, IFN-y production, cytotoxicity, and memory formation.

\section{The Blockade Of Tumor-derived Snkg2dls Recuperates Anti- tumor Function Of T Cells}

Lastly, we assessed the impact of NKG2D receptor expression on T cell function. Remarkably, the combination of anti-MICA/B and ULPB2, 3 resulted in improved expression of NKG2D receptor on CD $8^{+} \mathrm{T}$ cell, suggesting a cooperative therapeutic effect of targeting all kind of soluble NKG2D ligands (Fig. 4A, $B)$. Furthermore, to investigate whether targeting the soluble NKG2D ligand could recuperate CD $8+T$ cell function, we utilized single and combined soluble NKG2D ligands neutralized antibody in soluble NKG2DL and T cells co-cultured system. Single-agent therapy with anti-ULBP2 significantly increased percentages of IFN- $\gamma^{+}, \mathrm{Ki} 67^{+}$and $\mathrm{CD} 107 \mathrm{a}^{+}$in $\mathrm{CD} 8^{+} \mathrm{T}$ cell, whereas anti-MICA/B or anti-ULBP3 alone did not present such a significant impact (Fig. 4C, D). Combined therapy resulted in further significantly increased responsiveness of $C D 8^{+} T$ cells as compared to each monotherapy and antibody targeting tumor-derived soluble NKG2D ligands recuperate T cell anti-tumor function (Fig. 4C, D). Therefore, the 
blockade of tumor-derived sNKG2DLs recuperates anti-tumor function of T cells, supplying a promising strategy for tumor immunotherapy.

\section{Discussion}

Immune cell-based anti-tumor therapy has achieved incredible clinical outcomes, while tumor-derived NKG2D ligands as a key factor are involved in the immunosuppression to help tumor cells escape from anti-tumor immunity. Thus, the underlying mechanisms need be further investigation. In this study, with clinical efficacy grouped analysis and external T cell functional analysis, we found MICA/B and ULBP-2 as the main SNKG2DLs derived from neuroblastoma, and they were negatively correlated with clinical treatment outcome and the alteration of immune pattern. These NKG2DLs can be shed by ADAM10 and ADAM17, and impair anti-tumor activity of $C D 8^{+} T$ cell. Importantly, the soluble NKG2DL neutralized antibody could recuperate $T$ cell function greatly, which open a new path for anti-tumor immunotherapy.

Immune escape of NK cell immune surveillance during tumor progression results from the release of soluble ligands from the tumor cell surface by shedding. These soluble molecules (sNKG2D-L) passively block NKG2D and induce receptor internalization to down-regulate NKG2D on the surface of NK cells and thereby impair NK cell function [17]. While, there is still not completely known about the relevance of NKG2D ligands in neuroblastoma and NKG2D on T cell. Previous study has identified MICA/B in most NB patient sera[18]. But based on sera of NB patients, we found both MICA/B and ULBP-2 were the majority of released soluble NKG2D ligands in NB patients, characterized by high concentration of MICA/B. The expression of MICA/B and ULBP1, 2, 3 could be found in most NB tumor cell lines, while high levels of sMICA/B, sULBP-2 and sULBP-3 were detected in supernatant of SH-Y5Y, SK-N-BE(2), and LA-N-5, and low levels of sULBP-1 was showed in all NB cell lines. Meanwhile, it has been reported that serum levels of SNKG2D-L correlate with a poor prognosis and are used as prognostic markers in some solid tumors[19]. Here, we found the similar phenomenon that soluble ligands for NKG2D would correlate with an immunosuppressive microenvironment and with a poor prognosis in neuroblastoma patients.

A number of proteases have been involved in the cleavage of NKG2D ligands, including different members of metalloprotease family namely matrix metalloproteases and ADAMs [20]. Among members of ADAM families, the relative contribution of ADAM10 and ADAM17 in the NKG2D ligands cleavage is still undefined [21, 22]. In glioblastoma initiating cells, roles for ADAM10 and ADAM17 have been demonstrated in the shedding of ULBP-2 molecules [23]. In Hodgkin lymphoma cells, ADAM10 showed higher specificity for shedding of MICA/B and ULBP-3 over ADAM17 [24]. According to our research, both ADAM10 and ADAM17 are involved in the cleavage of MICA/B, ULBP2 and ULBP3 from neuroblastomas.

SNKG2D-L has been to be highly immune-suppressive via perturbing NK cell peripheral maintenance and function [25], blocking SNKG2D-L release in preclinical models enhances NK cell function [26]. Other research also reported impairing TCR/CD3 signaling by caspase-dependent destabilization of CD3ろ [27], and facilitating the expansion of MDSCs and tumor-associated macrophages [28] in SNKG2D-L immunesuppressive mechanisms. Consistently, we found soluble MICA/B and ULBP-2 selectively downregulate 
NKG2D on $\mathrm{CD}^{+} \mathrm{T}$ cells and impaired the proliferation, IFN-y production, cytotoxicity of $\mathrm{CD}^{+} \mathrm{T}$ cells. Corresponding to NKG2D receptor reported as a costimulatory molecule and responsible for memory formation in $\mathrm{CD}^{+} \mathrm{T}$ cells[29], we found SNKG2D-L significantly downregulate percentage of activated $\mathrm{CD} 8^{+} \mathrm{T}$ cell $\left(\mathrm{CD} 69^{+} \mathrm{CD} 25^{+}\right)$and $\mathrm{CD} 45 \mathrm{RO}^{+} \mathrm{CCR}^{+}$memory $\mathrm{CD} 8^{+} \mathrm{T}$ cells.

In humans, NKG2D is abundantly expressed on cytotoxic NK cells and CD8 ${ }^{+} \mathrm{T}$ cells and is also detectable on $\mathrm{CD} 4^{+} \mathrm{T}$ cell subsets, $\mathrm{T}$ cells, NKT cells, and Tregs [5]. The immunotherapeutic approaches currently adopted for high-risk patients are based on the infusion of NK cells, as support of consolidation therapy in addition to standard treatments, or CAR-T cells directed against neuroblastoma associated antigens (e.g., GD2), and future perspectives of adoptive cell therapies represented by T lymphocytes and CAR NK cells $[13,30,31]$. sNKG2D-L induced immune-suppressive effects can directly and indirectly impair NK and $\mathrm{CD} 8^{+} \mathrm{T}$ cell activation, and thus negatively impact the response to immunotherapeutic approaches based on these cells[32]. Our data proved that the clearance of sNKG2D-L rescues CD8 ${ }^{+} T$ cell function and enhances the sensitivity to anti-tumor effect in neuroblastoma. These profound therapeutic effects elicited by clearing SNKG2D-L could potentiate the $C D 8^{+} T$ cell response, in particular CAR-T-cell therapy.

\section{Statements And Declarations}

Funding: This study received financial support from National Natural Science Foundation of China (81771633 and 81572324)

Competing interests: The authors declare no competing interests.

Ethics approval: This study was approved by the Institute Research Ethics Committee at the Children's Hospital of Fudan University. Informed consent was acquired from every patient's legal guardians (NO.2020238)

\section{Acknowledgements}

This study received financial support from National Natural Science Foundation of China (grant nos. 81771633 and 81572324$)$

\section{Author contributions}

$\mathrm{KD}$ and $\mathrm{FL}$ designed the study. YZ collected the data and performed experiments. YZ and FL analyzed and interpreted the data. $Y Z$ and FL wrote the main manuscript text. DK and FL were involved in critical reviewing of the manuscript. All authors reviewed and approved the final manuscript.

\section{Data availability}

The data sets used and/or analyzed during current study are available from the corresponding author upon reasonable request. 


\section{References}

1. Maris JM (2010) Recent advances in neuroblastoma. N Engl J Med 362:2202-2211. https://doi.org/10.1056/NEJMra0804577

2. Park JA, Cheung N-KV (2020) Targets and Antibody Formats for Immunotherapy of Neuroblastoma. J Clin Oncol Off J Am Soc Clin Oncol 38:1836-1848. https://doi.org/10.1200/JC0.19.01410

3. Janoueix-Lerosey I, Schleiermacher G, Delattre O (2010) Molecular pathogenesis of peripheral neuroblastic tumors. Oncogene 29:1566-1579. https://doi.org/10.1038/onc.2009.518

4. López-Soto A, Huergo-Zapico L, Acebes-Huerta A, et al (2015) NKG2D signaling in cancer immunosurveillance. Int J Cancer 136:1741-1750. https://doi.org/10.1002/ijc.28775

5. Bauer S, Groh V, Wu J, et al (1999) Activation of NK cells and T cells by NKG2D, a receptor for stressinducible MICA. Science 285:727-729. https://doi.org/10.1126/science.285.5428.727

6. Prajapati K, Perez C, Rojas LBP, et al (2018) Functions of NKG2D in CD8 + T cells: an opportunity for immunotherapy. Cell Mol Immunol 15:470-479. https://doi.org/10.1038/cmi.2017.161

7. Mujal AM, Delconte RB, Sun JC (2021) Natural Killer Cells: From Innate to Adaptive Features. Annu Rev Immunol 39:417-447. https://doi.org/10.1146/annurev-immunol-101819-074948

8. Prajapati K, Perez C, Rojas LBP, et al (2018) Functions of NKG2D in CD8 + T cells: an opportunity for immunotherapy. Cell Mol Immunol 15:470-479. https://doi.org/10.1038/cmi.2017.161

9. Raulet DH (2003) Roles of the NKG2D immunoreceptor and its ligands. Nat Rev Immunol 3:781-790. https://doi.org/10.1038/nri1199

10. Liu H, Wang S, Xin J, et al (2019) Role of NKG2D and its ligands in cancer immunotherapy. Am J Cancer Res 9:2064-2078

11. Pistoia V, Morandi F, Bianchi G, et al (2013) Immunosuppressive microenvironment in neuroblastoma. Front Oncol 3:167. https://doi.org/10.3389/fonc.2013.00167

12. Fuertes MB, Domaica Cl, Zwirner NW (2021) Leveraging NKG2D Ligands in Immuno-Oncology. Front Immunol 12:713158. https://doi.org/10.3389/fimmu.2021.713158

13. Morandi F, Sabatini F, Podestà M, Airoldi I (2021) Immunotherapeutic Strategies for Neuroblastoma: Present, Past and Future. Vaccines 9:43. https://doi.org/10.3390/vaccines9010043

14. Park JR, Digiusto DL, Slovak M, et al (2007) Adoptive transfer of chimeric antigen receptor re-directed cytolytic T lymphocyte clones in patients with neuroblastoma. Mol Ther J Am Soc Gene Ther 15:825-833. https://doi.org/10.1038/sj.mt.6300104

15. Pule MA, Savoldo B, Myers GD, et al (2008) Virus-specific T cells engineered to coexpress tumorspecific receptors: persistence and antitumor activity in individuals with neuroblastoma. Nat Med 14:1264-1270. https://doi.org/10.1038/nm.1882

16. Kailayangiri S, Altvater B, Spurny C, et al (2017) Targeting Ewing sarcoma with activated and GD2specific chimeric antigen receptor-engineered human NK cells induces upregulation of immuneinhibitory HLA-G. Oncoimmunology 6:e1250050. https://doi.org/10.1080/2162402X.2016.1250050 
17. Zingoni A, Molfetta R, Fionda C, et al (2018) NKG2D and Its Ligands: "One for All, All for One." Front Immunol 9:476. https://doi.org/10.3389/fimmu.2018.00476

18. Raffaghello L, Prigione I, Airoldi I, et al (2004) Downregulation and/or release of NKG2D ligands as immune evasion strategy of human neuroblastoma. Neoplasia N Y N 6:558-568. https://doi.org/10.1593/neo.04316

19. Paschen A, Sucker A, Hill B, et al (2009) Differential clinical significance of individual NKG2D ligands in melanoma: soluble ULBP2 as an indicator of poor prognosis superior to S100B. Clin Cancer Res Off J Am Assoc Cancer Res 15:5208-5215. https://doi.org/10.1158/1078-0432.CCR-09-0886

20. Zingoni A, Vulpis E, Loconte L, Santoni A (2020) NKG2D Ligand Shedding in Response to Stress: Role of ADAM10. Front Immunol 11:447. https://doi.org/10.3389/fimmu.2020.00447

21. Waldhauer I, Steinle A (2006) Proteolytic release of soluble UL16-binding protein 2 from tumor cells. Cancer Res 66:2520-2526. https://doi.org/10.1158/0008-5472.CAN-05-2520

22. Waldhauer I, Goehlsdorf D, Gieseke F, et al (2008) Tumor-associated MICA is shed by ADAM proteases. Cancer Res 68:6368-6376. https://doi.org/10.1158/0008-5472.CAN-07-6768

23. Wolpert F, Tritschler I, Steinle A, et al (2014) A disintegrin and metalloproteinases 10 and 17 modulate the immunogenicity of glioblastoma-initiating cells. Neuro-Oncol 16:382-391. https://doi.org/10.1093/neuonc/not232

24. Tosetti F, Venè R, Camodeca $C$, et al (2018) Specific ADAM10 inhibitors localize in exosome-like vesicles released by Hodgkin lymphoma and stromal cells and prevent sheddase activity carried to bystander cells. Oncoimmunology 7:e1421889. https://doi.org/10.1080/2162402X.2017.1421889

25. Liu G, Lu S, Wang X, et al (2013) Perturbation of NK cell peripheral homeostasis accelerates prostate carcinoma metastasis. J Clin Invest 123:4410-4422. https://doi.org/10.1172/JCl69369

26. Ferrari de Andrade L, Tay RE, Pan D, et al (2018) Antibody-mediated inhibition of MICA and MICB shedding promotes NK cell-driven tumor immunity. Science 359:1537-1542. https://doi.org/10.1126/science.aao0505

27. Zhang J, Liu D, Li G, et al (2017) Antibody-mediated neutralization of soluble MIC significantly enhances CTLA4 blockade therapy. Sci Adv 3:e1602133. https://doi.org/10.1126/sciadv.1602133

28. Xiao G, Wang X, Sheng J, et al (2015) Soluble NKG2D ligand promotes MDSC expansion and skews macrophage to the alternatively activated phenotype. J Hematol OncolJ Hematol Oncol 8:13. https://doi.org/10.1186/s13045-015-0110-z

29. Perez C, Prajapati K, Burke B, et al (2019) NKG2D signaling certifies effector CD8 T cells for memory formation. J Immunother Cancer 7:48. https://doi.org/10.1186/s40425-019-0531-2

30. Quamine AE, Olsen MR, Cho MM, Capitini CM (2021) Approaches to Enhance Natural Killer CellBased Immunotherapy for Pediatric Solid Tumors. Cancers 13:2796. https://doi.org/10.3390/cancers13112796

31. Liu S, Galat V, Galat Y, et al (2021) NK cell-based cancer immunotherapy: from basic biology to clinical development. J Hematol OncolJ Hematol Oncol 14:7. https://doi.org/10.1186/s13045-02001014-w 
32. Basher F, Dhar P, Wang X, et al (2020) Antibody targeting tumor-derived soluble NKG2D ligand SMIC reprograms NK cell homeostatic survival and function and enhances melanoma response to PDL1 blockade therapy. J Hematol OncolJ Hematol Oncol 13:74. https://doi.org/10.1186/s13045-02000896-0

\section{Figures}
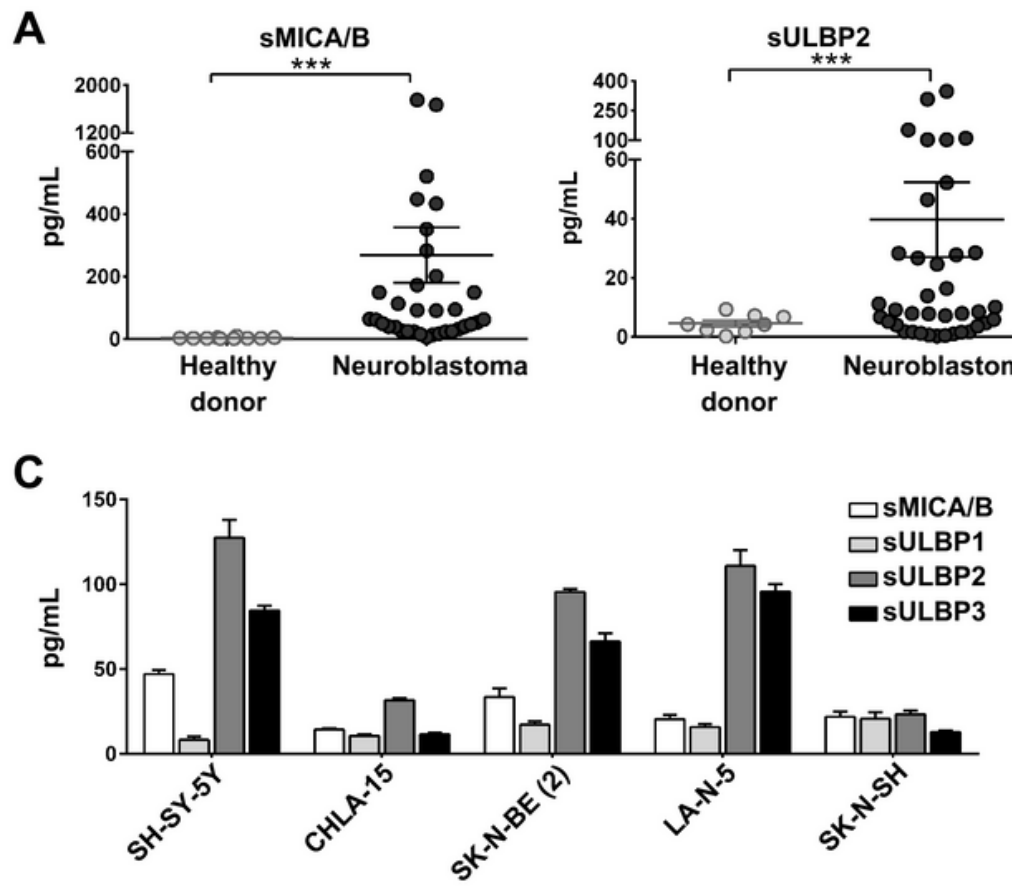

$\mathbf{E}$
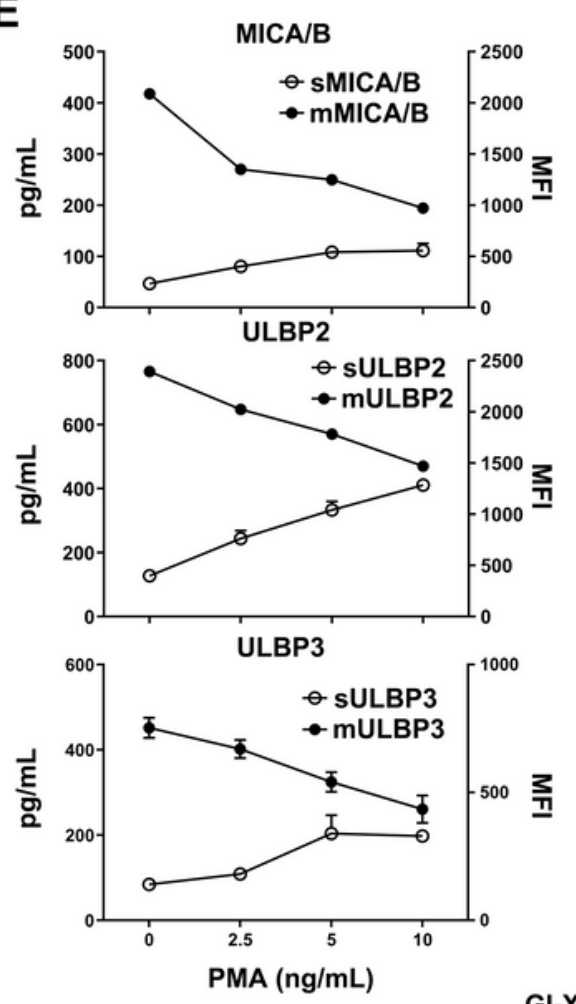

F
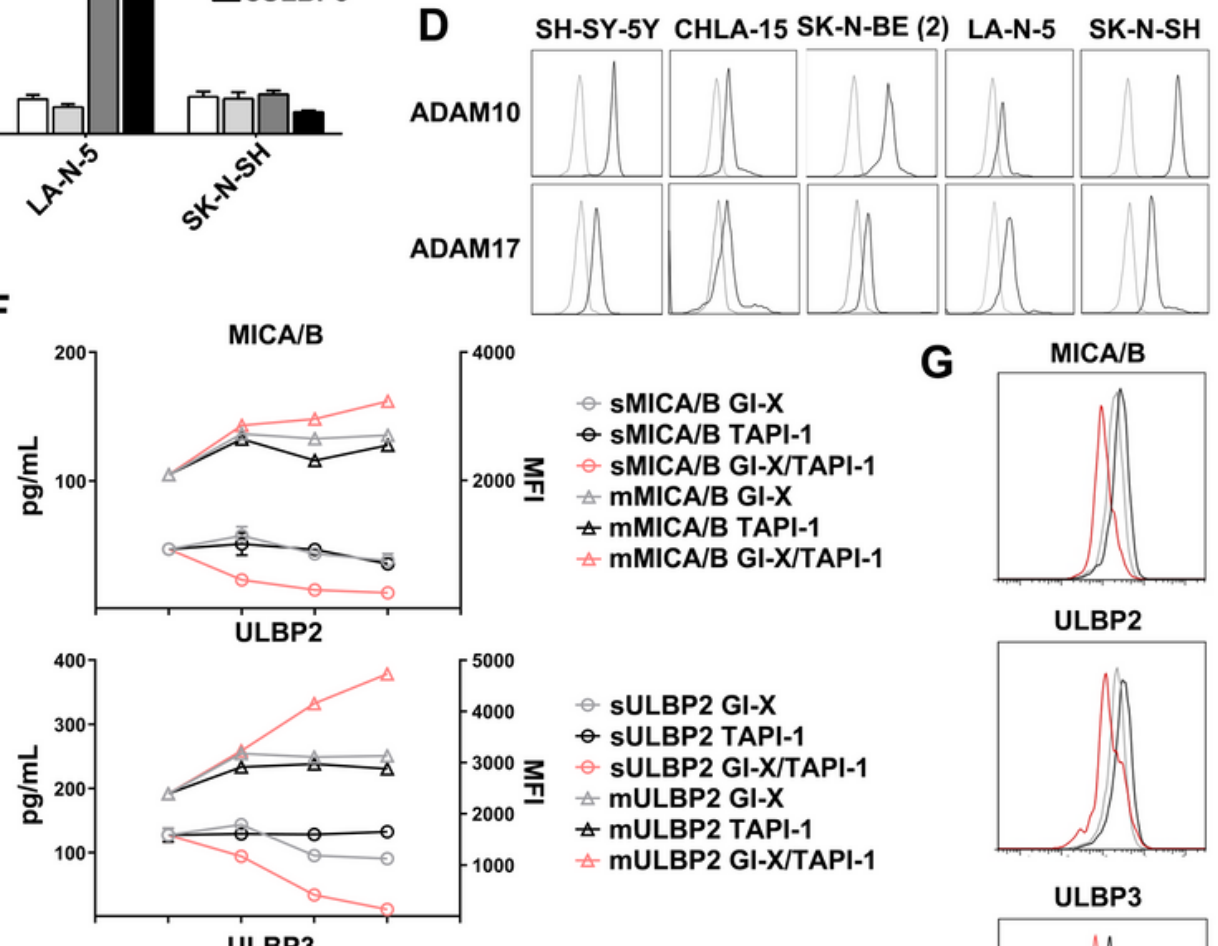

ULBP3

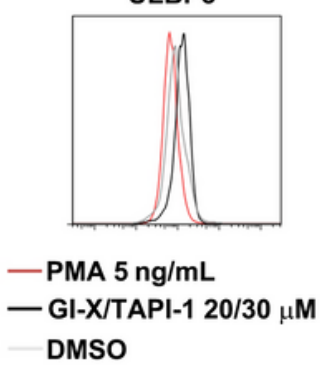




\section{Figure 1}

Tumor-derived sNKG2DLs are shed by ADAM10 and ADAM17. (A) Detection of SMICA and SULBP-2 by ELISA in serum samples from 35 neuroblastoma patients and 10 health donor. (B) MICA/B and ULBP1 3 expressions on neuroblastoma cell lines were assessed using flow cytometry. (C) Two days later, sMICA and sULBP1 3 production in culture supernatant was collected and measured by ELISA. (D) Surface expression of ADAM10 and ADAM17 in neuroblastoma cell lines were analyzed by flow cytometry. (E-G) Neuroblastoma cell lines cells were pretreated with the ADAM10/ ADAM17 promotor PMA, ADAM10 inhibitor GI-X, or ADAM17 inhibitor TAPI-1 for 24 hours. Cell surface MFI of MICA/B and ULBP2 was detected by flow cytometry. Levels of SMICA/B and SULBP2 in culture supernatant were analyzed by ELISA. Data were shown as mean \pm SEM. $P$ values were determined by unpaired Student's $t$ test in $\mathbf{A}$. *** $P$ $<0.01$. 
A

SMICA/B

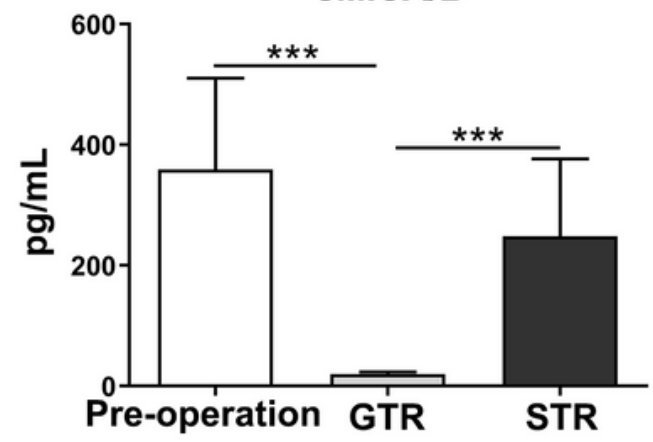

B

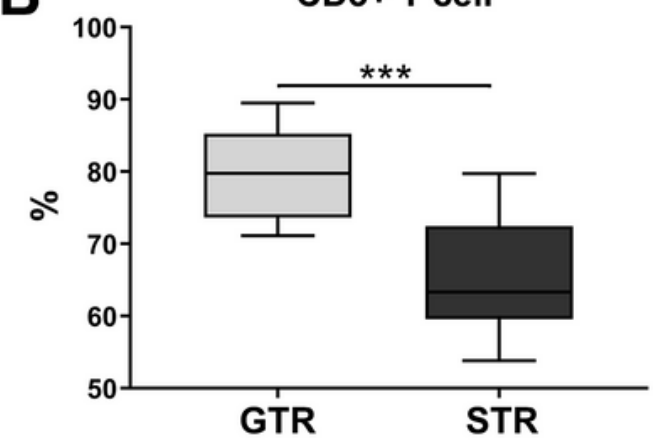

C

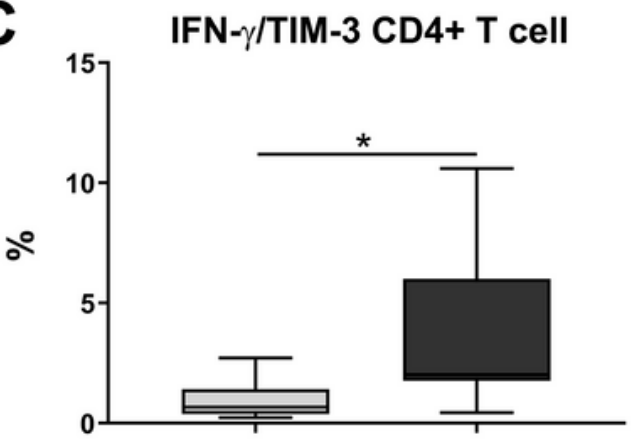

IFN- $\gamma /$ TIM-3 CD8+ T cell

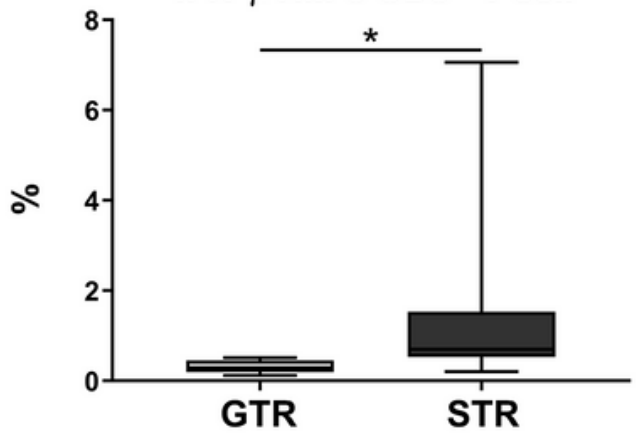

sULBP2

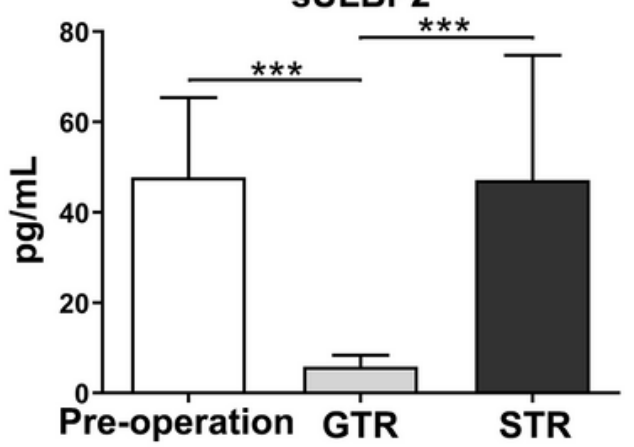

Foxp3+CD4+ Treg

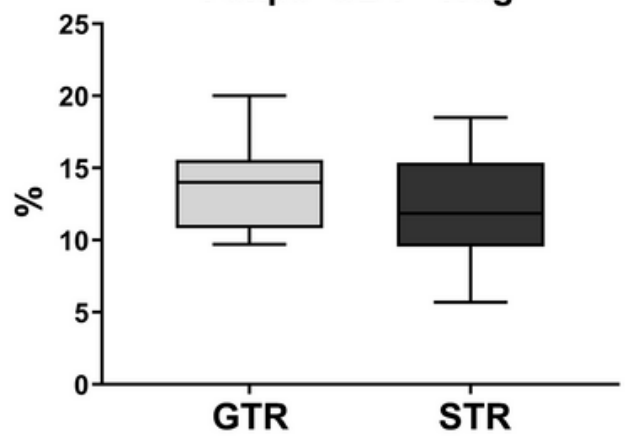

IFN- $\gamma /$ PD-1 CD4+ T cell

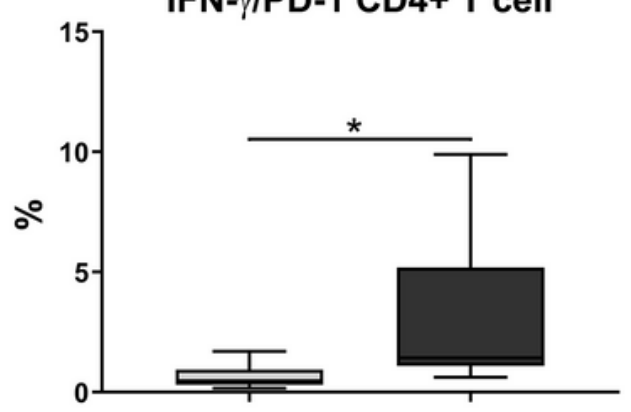

IFN- $\gamma /$ PD-1 CD8+ T cell

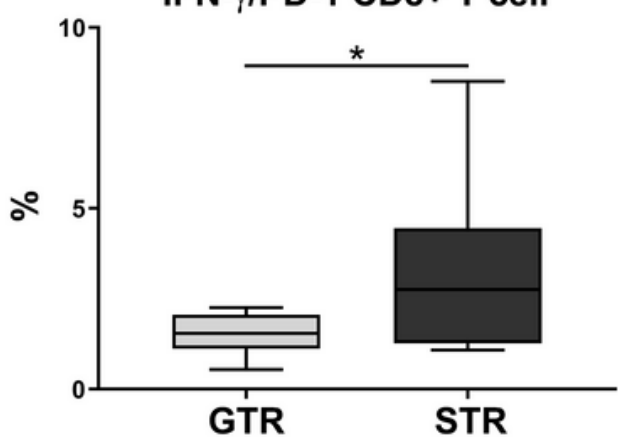

Figure 2

SNKG2DLs are negatively correlated with patient prognosis and the low-exhausted phenotype of T cells. (A) sMICA/B and sULBP-2 levels in serum samples from pre-operational patient, GTR and STR patients were measured by ELISA. (B) Percentages of T cell and Treg cell derived from PBMCs of GTR and STR patients were determined using flow cytometry. (C) The expression of inhibitory receptors PD-1 and TIM-3 by IFN- $\gamma^{+} \mathrm{CD} 4^{+}$or $\mathrm{CD} 8^{+} \mathrm{T}$ cells derived from PBMCs of GTR and STR patients were determined using flow 
cytometry. Data were shown as mean \pm SEM. $P$ values were determined by one-way ANOVA in $A$ and unpaired Student's $t$ test in B and C. $* P<0.05 ; * \star \star P<0.001$.
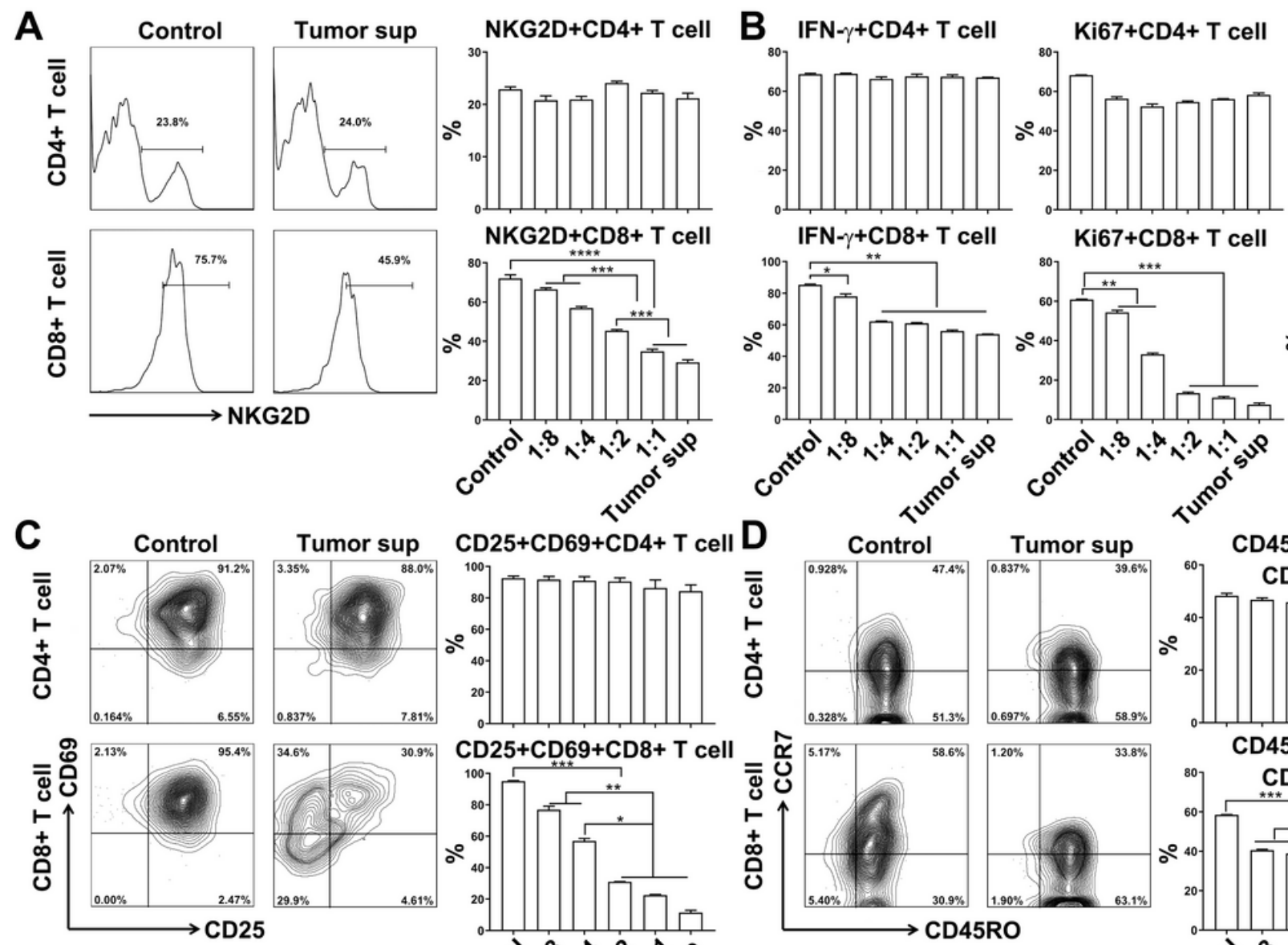

Ki67+CD8+ T cell

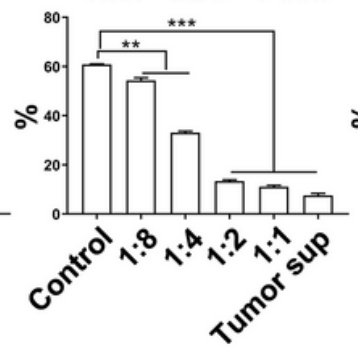

CD107a+CD8+ T cell

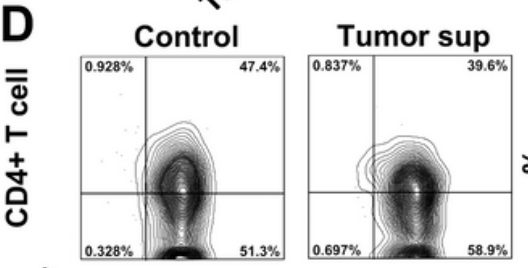

CD45RO+CCR7+
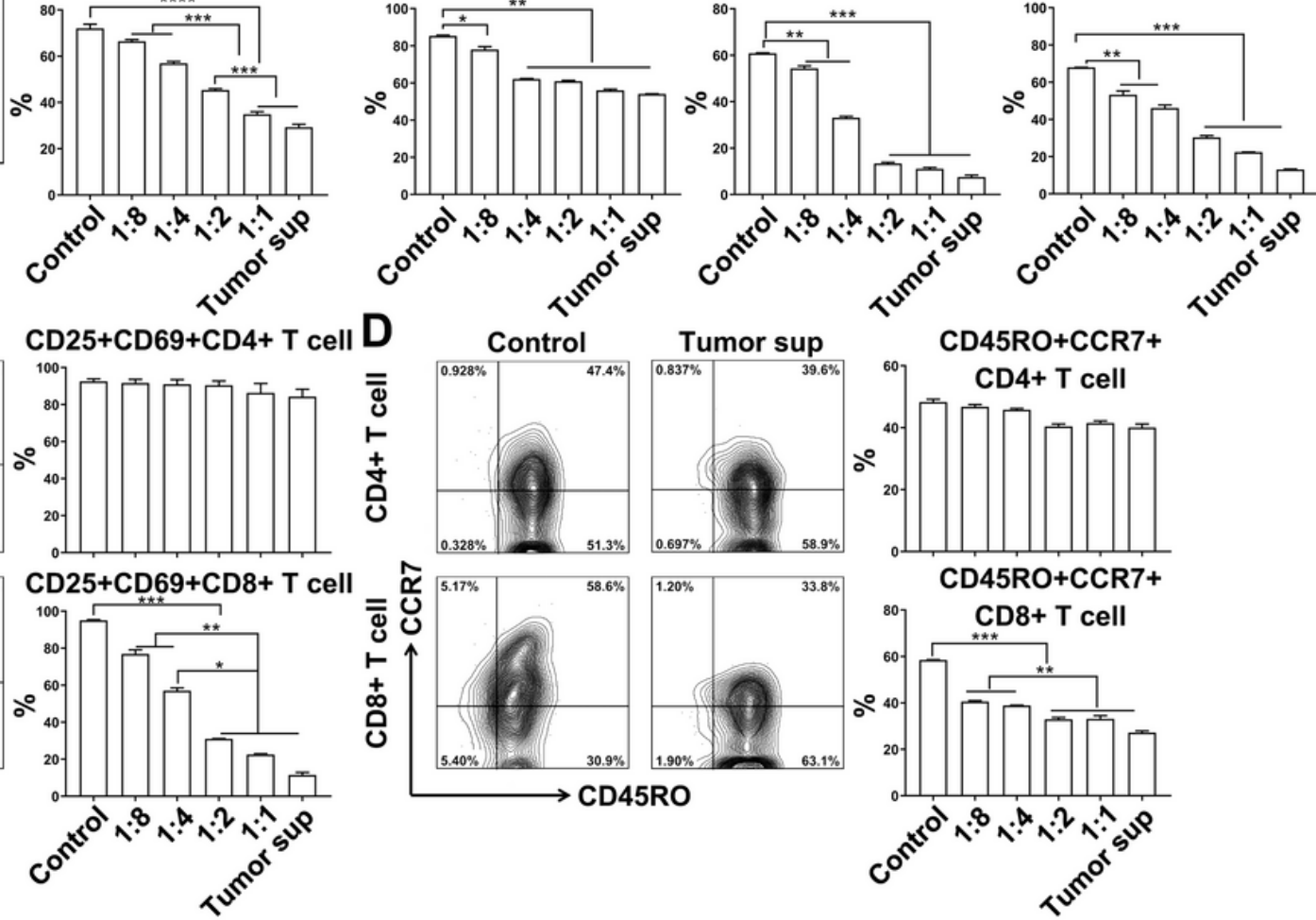

Figure 3

sNKG2DL impair CD8+ T cell function and memory formation. (A) Human T cells derived from health donors were activated and co-cultured with different concentrations of tumor cell culture supernatant for 12 hours. The NKG2D expression was detected by FACS. (B) Intracellular IFN-y production, Ki67 expression and CD107a translocation of CD8 ${ }^{+} \mathrm{T}$ cells were detected by flow cytometry. (C, D) Surface markers (CD25, CD69, CD45RO, and CCR7) of CD8 ${ }^{+} \mathrm{T}$ cells were also analyzed by FACS. Data were shown as mean \pm SEM. $P$ values were determined by one-way ANOVA in A-D. ${ }^{\star} P<0.05 ; \star \star \star P<0.01 ; * \star \star P<0.001$; $\star \star \star \star ~ P<0.0001$. 


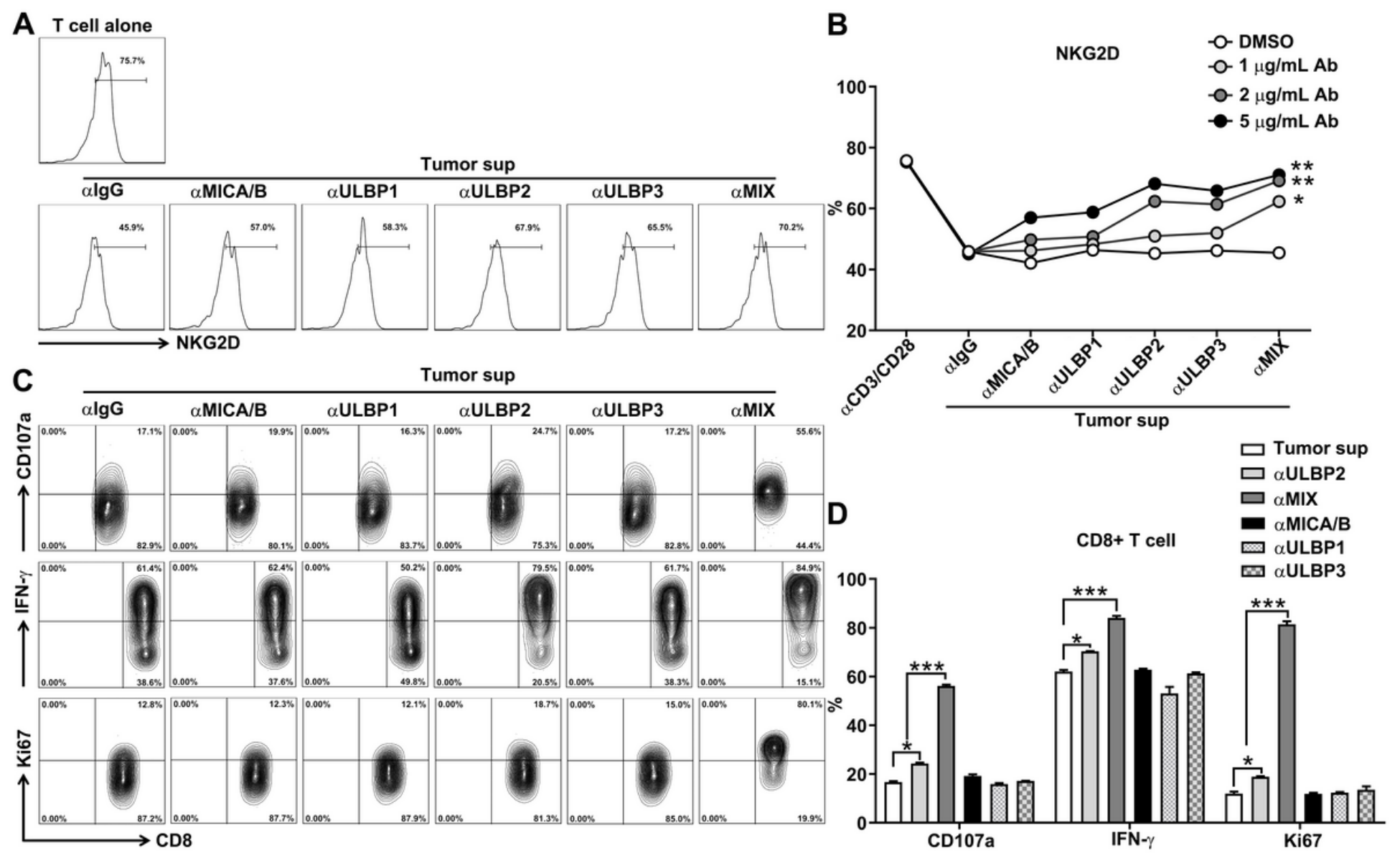

Figure 4

The blockade of tumor-derived sNKG2DLs recuperates anti-tumor function of T cells. (A, B) Human T cells derived from health donors were activated and co-cultured with tumor cell culture supernatant for 12 hours. Then, these $T$ cells were treated with single and combined soluble NKG2D ligands neutralized antibody (anti-MICA/B and/or ULBP1 3). After 2 hours, cell-surface expression of NKG2D was analyzed on $\mathrm{CD}^{+} \mathrm{T}$ cells by FACS. (C, D) After additional 10 hours, intracellular IFN-y production, Ki67 expression and CD107a translocation of CD $8^{+} T$ cells were detected by flow cytometry. Data were shown as mean \pm

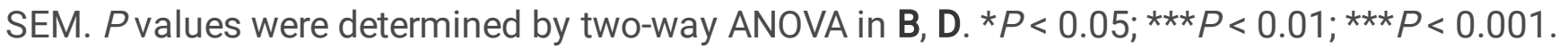

